\title{
Responses of Renal Mechanoreceptors to Vasopressor Substances
}

\author{
Hideo Ueda, M.D., Yasumi Uchida, M.D., and \\ Kazuaki KamisaKa, M.D.
}

\section{SUMMARY}

Responses of the renal mechanoreceptors to vasopressor agents were examined in mongrel dogs anesthetized with pentobarbital sodium. On injecting the agents, intrarenal pressure fell at first, then returned gradually to control level and overshot thereafter. Renal venous pressure, however, was not always changed in parallel with intrarenal pressure. The frequency of the impulses of low voltage, spontaneously discharging and slowly adapting units increased transiently at the onset of the fall in the intrarenal pressure, then decreased or disappeared. Thereafter, the frequency increased markedly with the return or overshooting of the pressure. The impulses of higher voltage and threshold, not spontaneously discharging and rapidly adapting units appeared transiently at the fall or return of the pressure. These findings are similar to those obtained in the previous studies in which the intrarenal pressure was altered mechanically to different direction from either renal arterial or venous pressure.

It is concluded that the mechanoreceptors were excited by the vasopressor agents indirectly through the alterations in the intrarenal pressure.

\section{Additional Indexing Words:}

Two types of afferent impulses

Renal venous and intrarenal pressure Noradrenalin Adrenalin Methoxamine

Angiotensin II

Serotonin

$\mathrm{I}^{\mathrm{N}}$ $\mathrm{N}$ the renal nerve, there are the affcrent impulses originating from the renal mechanoreceptors; some are of low voltage, are spontaneously discharging and adapt slowly (type A). Others, of higher voltage and threshold, do not discharge spontaneously and adapt rapidly (type B). ${ }^{6}$ ) It has hitherto been clarified to some extent what responses these receptors show to the mechanically induced alterations in renal hemodynamics. ${ }^{13-5)}$ However, it has not been settled whether the receptors are excited directly by the vasopressor agents and what responses they show to the agents. ${ }^{2)}$, ( The present investigations were undertaken to elucidate the mode of excitation of the receptors after the administration of different vasopressor agents.

From the Second Department of Internal Medicine, Faculty of Medicine, University of Tokyo, Tokyo.

Received for publication October 13, 1970. 


\section{Materials and Methods}

Twenty-seven mongrel dogs weighing from 9 to $13 \mathrm{Kg}$. were used in the present studies, The dogs were anesthetized with pentobarbital sodium (35 to $40 \mathrm{mg}$. $/ \mathrm{Kg}$.), injected intravenously. The respiration was maintained with an endotracheal tube attached to a respirator. The dogs were immobilized on their right side. The left flank was opened and the retroperitoneum was resected to pull out the left kidney.

The renal nerves were dissected from their surrounding tissues and the cut peripheral end was lifted up onto bipolar electrodes. The afferent impulses in these fibers were amplified for display on an oscilloscope, and were photographed. At the same time, the impulses were employed to trigger a square wave generator, and the output was integrated by a pulse-integrator and recorded on a pen-oscillograph. The height of the integrated record indicate the number of the impulses per sec. The pressures were recorded by Statham strain gauges connected to the needles inserted into the renal cortex or medulla, the renal artery and to a catheter introduced through the spermatic vein into the left renal vein. The vasopressor agents were injected through a catheter in a cutaneous vein of the right forelimb. The doses of the agents injected were as follows: noradrenalin: 0.03 to $5.5 \mu \mathrm{g} . / \mathrm{Kg}$., angiotensin II : 0.006 to $0.6 \mu \mathrm{g} . / \mathrm{Kg}$., adrenalin : 0.029 to $2.4 \mu \mathrm{g} . / \mathrm{Kg}$, serotonin: 0.7 to $7 \mu \mathrm{g} . /$ $\mathrm{Kg}$, methoxamine: 0.015 to $1.5 \mu \mathrm{g} . / \mathrm{Kg}$.

\section{RESUlts}

Injections of the vasopressor agents caused an abrupt fall in intrarenal pressure followed by a gradual return to the control levels, and then usually an overshoot. The magnitude of the fall became larger and the slope of the fall became steeper with increasing doses. The renal venous pressure fell usually in parallel with the intrarenal pressure. However, recovery of the renal venous pressure and intrarenal pressure did not always occur in parallel. In several experiments, the intrarenal pressure returned to the control levels earlier than the venous pressure and overshooting was observed in the former but not in the latter.

\section{Responses of Type A impulses}

Following the administrations of noradrenalin, the rate of discharge of irregularly discharging units of type A decreased, and thereafter showed an increase with the return or overshooting of the pressure. In several experiments, a transient increase in the firing at the onset of the fall in the intrarenal pressure was observed. In several other experiments in which intrarenal pressure overshot markedly but in which the renal venous pressure did not, the impulse frequency increased markedly before and during the overshoot (Fig. 1).

Noradrenalin produced the initial excitatory phase in 2 , phase of decrease in 8 and the secondary excitatory phase in 14 of 18 trials in 6 dogs. The 
initial excitatory phase and the phase of decrease were observed in the trials in which the fall in the intrarenal pressure was marked. Fig. 2 shows an unusual instance in which the frequency of the impulses increased throughout the fall of the intrarenal pressure.

Adrenalin produced an initial increase in 3, a decrease in 5 and a second-

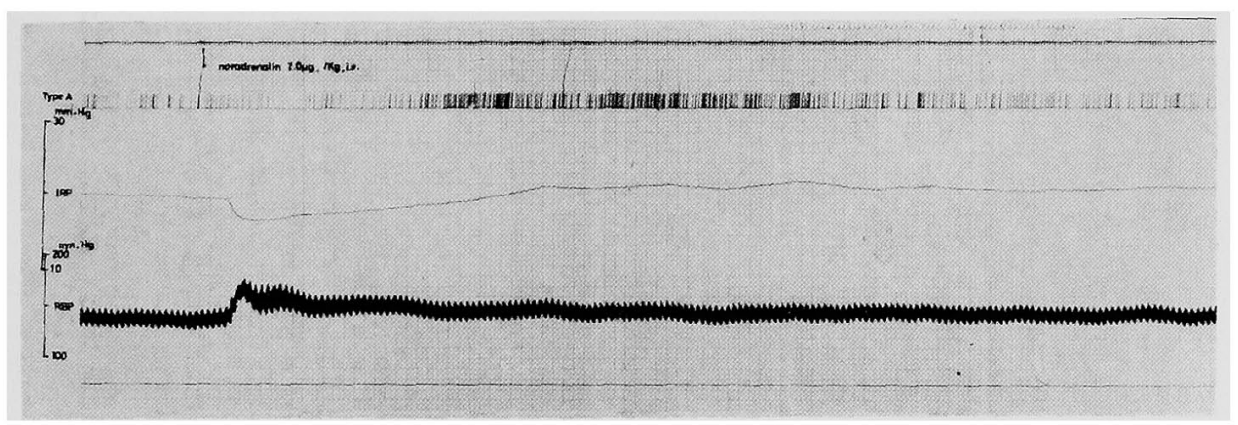

Fig. 1. Effects of intravenous injection of nocadrenalin on type A impulses.

RBP: renal blood pressure.

IRP: intrarenal pressure.

The top tracing indicates the time scale. Each segment indicates $1 \mathrm{sec}$.

The agent was injected at the point of the arrow.

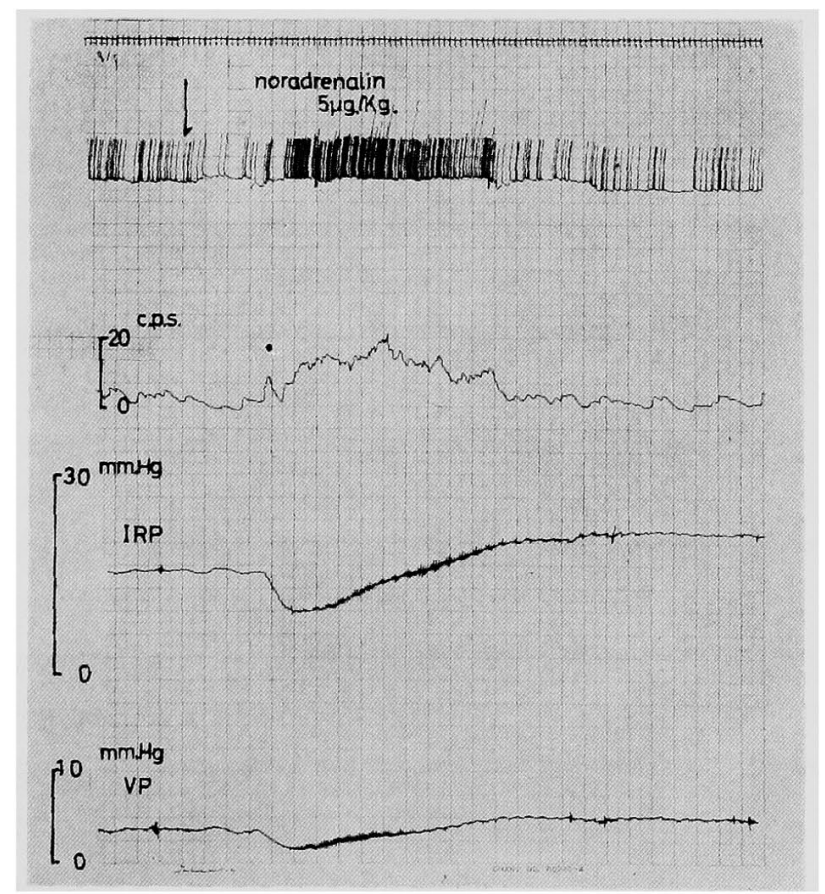

Fig. 2. Effects of noradrenalin on type A impulses. VP: venous pressure. The upper recording and the the lower recording were derived from the same preparation. Time constant for pulse integration in the lower is longer than that in the upper. 
ary increase in 4 of 8 trials in 3 dogs (Fig. 3). Methoxamine produced an initial increase in 3 , a decrease in 5 and a secondary increase in 4 of 8 trials in 4 dogs. Angiotensin II produced an initial increase in 9, a decrease in 11 and a secondary increase in all 14 trials in 6 dogs. Effects of angiotensin II on type A impulses of regularly discharging units was examined in one preparation. This unit responded to the agent in the same fashion as those of irregularly

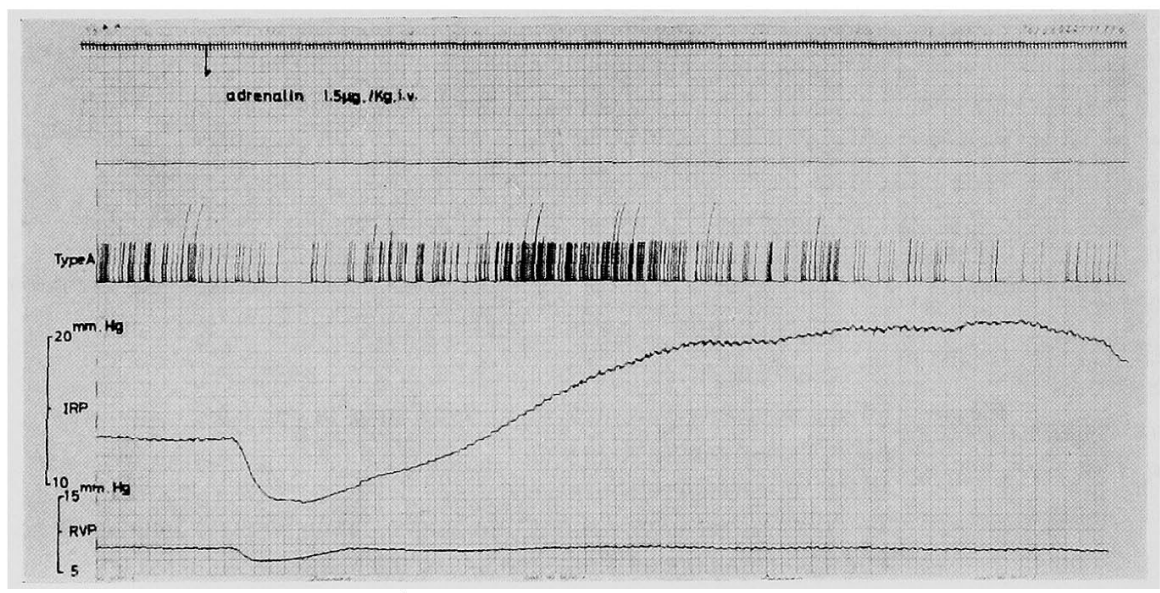

Fig. 3. Effects of adrenalin on type A impulses.

Time scale (the top) is 1 sec.

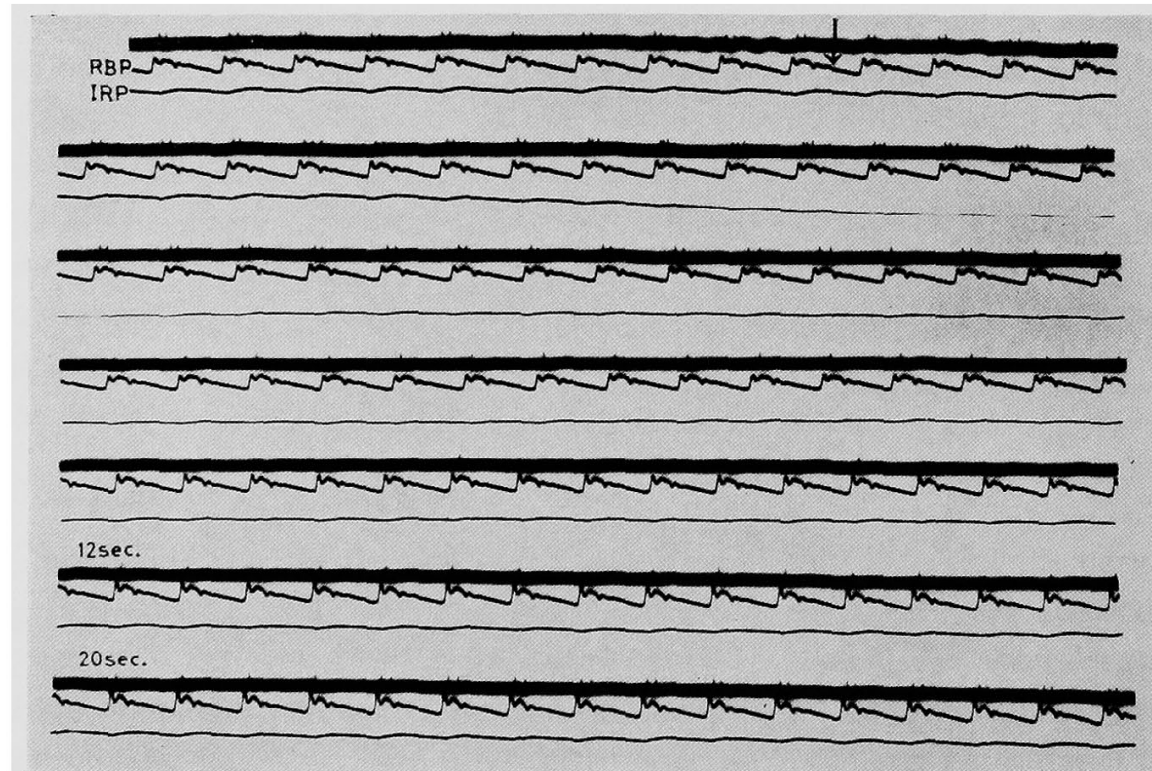

Fig. 4. Photographed recording. Angiotensin II, $0.46 \mu \mathrm{g} . / \mathrm{Kg}$., i.v. Note a transient increase in impulse frequency at the fall in the intrarenal pressure, and a following disappearance of the firing.

The 6 th and 7 th strips were obtained 12 and 20 sec. after the end of the 5 th strip, respectively. 
discharging units (Figs. 4 and 5).

Serotonin injections produced an initial increase in 2, a decrease in 2 and a secondary increase in 4 of 5 trials in one dog.

These alterations in impulse frequency were not observed when the fall in the intrarenal pressure was within $2 \mathrm{~mm} . \mathrm{Hg}$.

\section{Responses of Type B impulses}

Following the injections of noradrenalin, the impulses of this type appeared

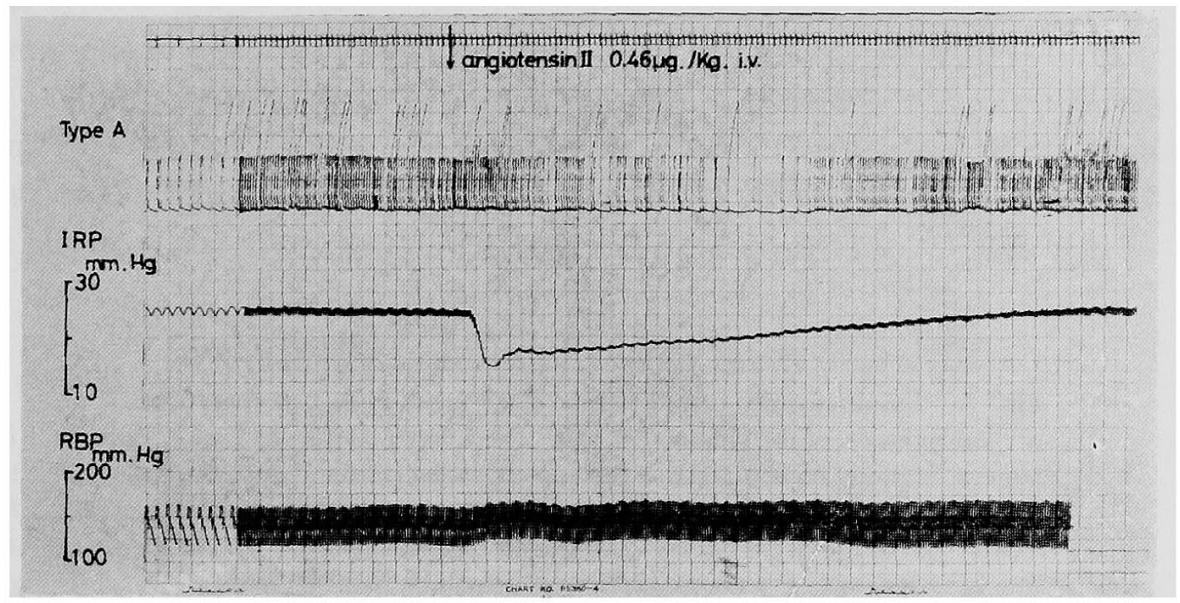

Fig. 5. Effects of angiotensin II on type A impulses of regularly discharging unit. This recording was obtained at the same time with those shown in Fig. 4. Each segment at the top indicates 1 sec.

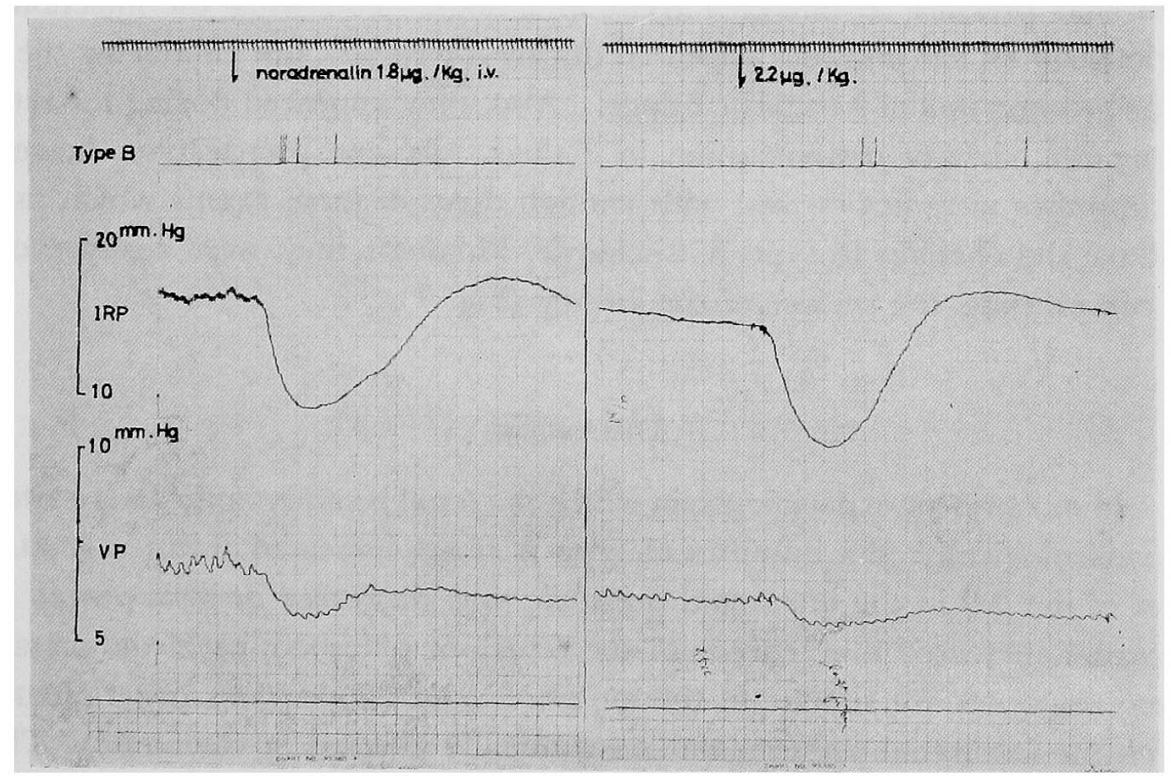

Fig. 6. Effects of noradrenalin on type B impulses. 


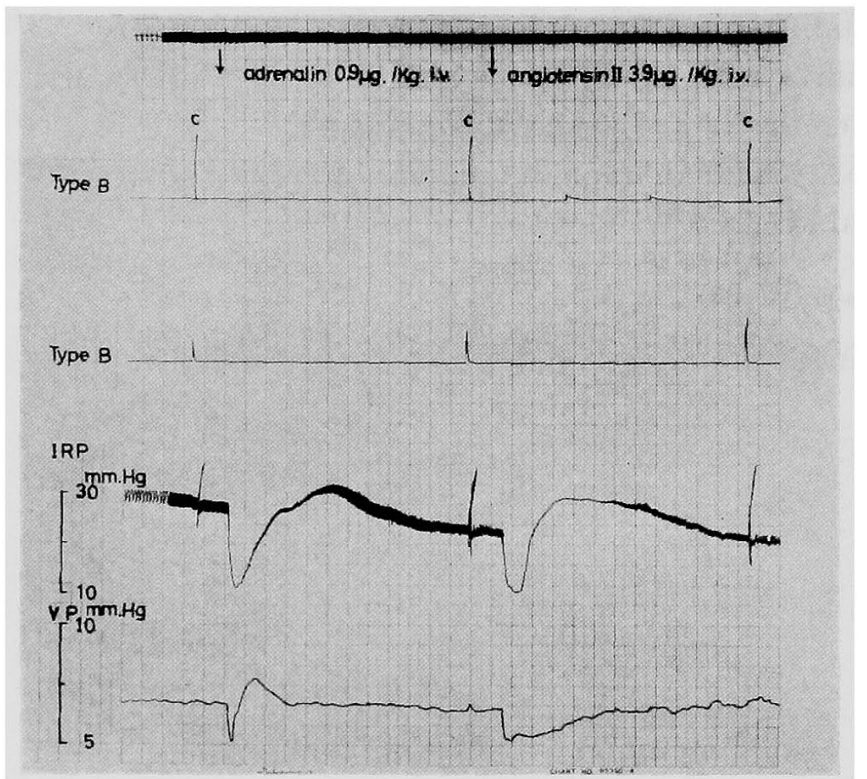

Fig. 7. Simultaneously recorded type B impulses from 2 preparations. c: compression of the kidney.

Note a response by compression but none by adrenalin and angiotensin II.

transiently at the fall in the intrarenal pressure in one and during the return or overshooting of the pressure in 6 of 11 trials in 4 dogs (Fig. 6).

Adrenalin caused an excitation of the units during the return of the intrarenal pressure in one of 4 trials in 2 dogs. Following the injections of angiotensin II, the impulses appeared during the return, but not during the fall of the pressure in 3 of 4 trials in 2 dogs. Also, they appeared during the return in one of 3 trials of methoxamine in 2 dogs. In one preparation, however, the impulses were not evoked with enough doses of these agents which might produce the changes in type A discharge, although they were easily evoked by compressing the surface of the kidney (Fig. 7).

\section{Discussion}

In our present experiments, in which the renal hemodynamics were altered pharmacologically, the impulses of type A fibers increased transiently at the onset of the fall in the intrarenal pressure, then decreased or disappeared and thereafter increased markedly with the return or overshooting of the pressure. These responses are similar to those observed in our previous experiments in which the intrarenal pressure was mechanically lowered or elevated. ${ }^{6)}$ These effects were related to the intrarenal pressure, but not to the venous and arterial 
pressures in the present experiments, as they were in our previous perfusion experiments in which the intrarenal pressure was varied mechanically. These findings support the possibility that type A impulses of both regularly and irregularly discharging units were altered by the vasopressor agents indirectly through the alterations in the intrarenal pressure.

Type $B$ impulses were evoked during the fall or return of the intrarenal pressure. The same responses were observed in our previous studies in which the intrarenal pressure was altered mechanically. Therefore, it is supposed that type B impulses were also evoked by the agents indirectly through the alterations in the intrarenal pressure.

Niijima noted that the renal afferent impulses were evoked by noradrenalin. ${ }^{1,2)}$ On the other hand, Pines observed that the frequency of the impulses was decreased by noradrenalin and adrenalin. ${ }^{3)}$ It is likely that Niijima tested the effects of the agents on type B fibers and Pines on type A fibers.

\section{REFERENCES}

1. Nijima, A. J. Jap. Soc. Physiol. $21: 477,1964$.

2. Niijima, A: J. Jap. Soc. Physiol. 27: 72, 1965.

3. Pines, Y. L.: Fiziol. Zh. SSSR, Sechenov. 45: 1139, 1959.

4. Tower, S. S.: J. Physiol. 21 : 477, 1964.

5. Ueda, H., Uchida, Y., and Kamisaka, K.: Jap. Heart J. 8: 597, 1967.

6. Uchida, Y., Kamisaka, K., and Ueda, H.: Jap. Heart J. 12: 233, 1971. 